\title{
The Convergence Time for Selfish Bin Packing*
}

\author{
György Dósa ${ }^{a}$ and Leah Epstein ${ }^{b}$
}

\begin{abstract}
In classic bin packing, the objective is to partition a set of $n$ items with positive rational sizes in $(0,1]$ into a minimum number of subsets called bins, such that the total size of the items of each bin at most 1 . We study a bin packing game where the cost of each bin is 1 , and given a valid packing of the items, each item has a cost associated with it, such that the items that are packed into a bin share its cost equally. We find tight bounds on the exact worst-case number of steps in processes of convergence to pure Nash equilibria. Those are processes that are given an arbitrary packing as an initial packing. As long as there exists an item that can reduce its cost by moving from its bin to another bin, in each step, a controller selects such an item and instructs it to perform such a beneficial move. The process converges when no further beneficial moves exist. The tight function of $n$ that we find is in $\Theta\left(n^{3 / 2}\right)$. This improves the previous bound of Ma et al. [14], who showed an upper bound of $O\left(n^{2}\right)$.
\end{abstract}

\section{Introduction}

We study a class of bin packing games, that are based on the well-known standard bin packing problem $[17,4,6,5]$, a basic combinatorial optimization problem. In this problem, a set of $n$ items $I=\{1,2, \ldots, n\}$ is given, where the size of item $t$, denoted by $s_{t}$, satisfies $0<s_{t} \leq 1$. The goal is to partition (or pack) the items into a minimum number of subsets or blocks. Each such block is packed into a unit capacity bin, and the load of a bin is defined to be the total size of items packed into it (and can never exceed 1). Here, we study bin packing from the point of view of algorithmic game theory.

We now define the game theoretical concepts required for the definition of the bin packing game. In a strategic game, there is a finite set of players, and a finite and non-empty set of strategies (or actions) that players can perform. Each player

\footnotetext{
*Supported by VKSZ_12-1-2013-0088 "Development of cloud based smart IT solutions by IBM Hungary in cooperation with the University of Pannonia" and by National Research, Development and Innovation Office - NKFIH under the grant SNN 116095

${ }^{a}$ Department of Mathematics, University of Pannonia, Veszprém, Hungary, E-mail: dosagy@almos . vein.hu

${ }^{b}$ Department of Mathematics, University of Haifa, Haifa, Israel. E-mail: lea@math.haifa.ac.il
} 
has to choose a strategy (possibly independently from other players). Each player has a cost for each one of the possible situations or outcomes, where an outcome is a possible set of strategies of all players, containing one strategy for each player. A classic form of a stable solution is a Nash equilibrium (NE) [21]. This is a kind of solution concept of a game with at least two players, where no player can decrease its cost by changing only its own strategy unilaterally. That is, if each player has chosen a (pure or mixed) strategy and no player can benefit by changing its strategy while the other players keep theirs unchanged, then the current set of strategy choices and the corresponding costs result in an outcome or solution that is a Nash equilibrium (NE). We are interested in pure Nash equilibria, where the actions of players are chosen in a deterministic way, and will discuss only this kind of NE.

Given an input for bin packing, the set of players are the items. The pure strategy of a player is the index of the bin into which it is packed (the number of possible bins is $n$, as this number of bins is always sufficient). We say that a bin $B \subseteq I$ is a valid bin if $\sum_{t \in B} s_{t} \leq 1$, that is, if its load does not exceed 1. Changing the strategy of an item means that it moves to be packed in a different (non-empty or empty) bin. For $0 \leq k \leq n$, we define a $k$-bin to be a bin that has exactly $k$ items, and a $k^{+}$-bin is a bin that has at least $k$ items. The cost of an item packed into a valid $k$-bin (for $k \geq 1$ ) is $\frac{1}{k}$. We let the cost of an item that is not packed into a valid bin be infinite. The deviation of an item $t$ packed in a $k_{1}$-bin $B_{1}$ (where $t$ is included in the number of items of $B_{1}$ ) to a $k_{2}$-bin $B_{2}$ (where $t$ is not included in the number of items of $B_{2}$ ) is beneficial if $s\left(B_{2}\right)+s_{t} \leq 1$ (since otherwise the cost of the item in the alternative bin is infinite) and $k_{2} \geq k_{1}$ (as otherwise its cost is not reduced by moving). The standard bin packing problem can be therefore seen as a class of games, where every input corresponds to a game. The uniform cost sharing rule is motivated by the well-known egalitarian or Shapley model in game theory, which was introduced in an algorithmic game-theoretic context by Anshelevich et al. [2]. Unlike other bin packing games, the variant with uniform sharing is a congestion game.

In this paper, we are interested in convergence processes. Such a process receives a set of items and a packing. The packing obviously corresponds to an outcome of the game whose players are those items. The process stops when it reaches a solution that is an NE. As long as it is not an NE, a step is performed. In each step, a controller selects an item that can benefit (reduce its cost) by moving to another bin, and instructs it to move from its current bin to a specified bin (where its cost will be smaller). In each step a single item moves and decreases its cost, while other items may be affected (those that were packed with the moving item will have larger costs, and those that were packed into the bin where it moved will have smaller costs). It is shown by Ma et al. [14] (who were the first to study the variant with equal sharing of the bin costs) that such a process always converges in $O\left(n^{2}\right)$ steps. This kind of games are in fact singleton congestion games [15, 16], but the number of resources has an exponential size in the number of players, and it is not given explicitly (these are all possible subsets of items that can be packed into a bin), so the convergence and existence of NE can be deduced from previous 
work on congestion games, but the polynomial time convergence cannot be deduced from those.

Bin packing in general, and more specifically bin packing games, have a number of applications [3, 9, 10]. Equal sharing is the simplest form of sharing, and it does not require prior information given by the players (who may or may not be truthful). Bin packing games where items share the cost of the bin proportionally (according to sizes) rather than equally was introduced by Bilò [3], who was the first to study the bin packing problem from this kind of game-theoretic perspective. $\mathrm{He}$ proved that every game in this class has an NE. He also proved that any such bin packing game converges to an NE after a finite (but possibly exponentially long) sequence of steps, starting from any initial configuration of the items. The time of convergence for this type of cost sharing was also studied in [19, 20]. Multiple papers studied the quality of $\mathrm{NE}$ and other types of equilibria $[3,9,10,7,1]$. Polynomial time algorithms that compute an NE for games with proportional cost sharing and for equal cost sharing are given in $[22,14,7]$. Note that the term "bin packing games" is used in the literature for a completely different type of games $[12,13,18]$, and there is recent interest in those games as well.

Our result. We show that the worst-case number of steps for convergence is $\Theta\left(n^{1.5}\right)$. The exact function expressing the worst-case number of steps is

$$
\frac{i(i+1)(i-1)}{3}+j-i j,
$$

where

$$
n=\frac{i(i+1)}{2}-j \text { for } \quad 0 \leq j \leq i-1 .
$$

We prove the lower bound by defining a sequence of steps, while the upper bound is proved using two potential functions, one of which is used in [14] and the other one is completely different. Interestingly, combining the two potential functions allows us to find a tight bound for any $n \geq 1$.

The maximal number of steps is achieved by a process that is not completely intuitive. The process is started with a packing where every item is packed into its own bin, and it ends with a packing where all items are packed into one bin. Moreover, it is obtained using items whose sizes are sufficiently small, such that all items can indeed be packed together. Those last properties of the initial packing and the final packing are natural as converting an arbitrary process to such a process, by reducing the sizes and adding steps in the beginning and at the end, obviously increases the number of steps. We show that the process of convergence can be split into two clear parts. In the first part, items migrate until a packing with a special structure which we call a staircase packing will be achieved. In such a packing, any non-empty bin contains different number of items, and these numbers (of items) are as small as possible (for example, if there are 17 items, there are five bins, containing one, two, three, five, and six items, respectively). After the staircase structure is built, it is destroyed again, to obtain fuller bins along time. The steps are carefully chosen such that the process is applied exactly in this way, and other steps are not performed. As we prove an exact bound (and not only an 
order of growth), the process is defined very carefully. An important part of the proof of the upper bound is Lemma 6, where we consider an interesting invariant holding until a staircase packing is created.

A preliminary version of this work appeared as [8].

\section{The exact convergence time}

In [14], processes of the following kind were studied. The process starts with an arbitrary packing, and in each step one item that can reduce its cost by moving to another bin is selected by a controller and is moved to another bin such that its cost becomes smaller. The number of steps for convergence was shown to be $O\left(n^{2}\right)$ [14]. In this section we find the exact worst case number of steps, which turns out to be $\Theta\left(n^{3 / 2}\right)$. Note that [19] showed using methods from [11] (where convergence for scheduling problems is studied) that for the case of proportional cost sharing, the number of steps can be exponential.

Given an integer $n \geq 1$, we let

$$
i=\min \{h \mid h(h+1) / 2 \geq n\} \quad \text { and } \quad j=i(i+1) / 2-n .
$$

Thus, $n=i(i+1) / 2-j$, where $i \geq 1$, and $0 \leq j \leq i-1$ (since $n>i(i-1) / 2=i(i+$ 1) $/ 2-i)$. Additionally, since $n \leq i(i+1) / 2<(i+1)^{2}$ and $n>i(i-1) / 2>(i-1)^{2} / 4$, we have $i>\sqrt{n}-1$ and $i<2 \sqrt{n}+1$, and thus $i=\Theta(\sqrt{n})$. We show that the maximum number of steps that can be performed for any set of items and initial configuration is exactly

$$
\nabla_{i, j}=\frac{i(i+1)(i-1)}{3}+j-i j .
$$

Note, that in case $i \geq 12$, the next inequalities are valid: $i-1 \geq i / 2, i / 6-1 \geq$ $i / 12$, and $i+1 \leq 2 i$. Thus, it can be verified that

$$
\nabla_{i, j} \leq \frac{i(i+1)(i-1)}{3}<i^{3}
$$

and

$$
\nabla_{i, j}>\frac{i^{3}}{6}-i^{2}=i^{2}(i / 6-1) \geq i^{3} / 12 .
$$

Thus, $\nabla_{i, j}=\Theta\left(n^{\frac{3}{2}}\right)$.

We start with the lower bound.

Lemma 1. For every positive integer $n$, there exists an input of $n$ items, for which there is an initial packing of these items, and a sequence of $\nabla_{i, j}$ steps that are performed until no additional steps can be done. 
Proof. Consider a set of $n$ items, each of size $\frac{1}{n}$, and an initial packing where each one of the items is packed in its own bin. Let a staircase packing be a packing where for every $1 \leq \eta \leq i, \eta \neq j$, there is exactly one bin with $\eta$ items.

We show using induction on $i$ that there exists a sequence of exactly $\Delta_{i, j}=$ $i(i+1)(i-1) / 6-j(j-1) / 2$ steps that results in a staircase packing. Note that for $i=1$, it holds that $j=0$, and thus $\Delta_{1,0}=0$. Otherwise $i \geq 2$, and in this case $j \geq 0$ and $0 \leq j(j-1) \leq(i-1)(i-2)$ hold. Thus we get the next chain of inequalities:

$i(i+1)(i-1)-3 j(j-1) \geq i(i+1)(i-1)-3(i-1)(i-2)=(i-1)\left(i^{2}-2 i+6\right)>0$

where we used the property $i^{2}-2 i+6 \geq 6$. First, we show the claim for the case $j=0$ (where $\left.\Delta_{i, 0}=i(i+1)(i-1) / 6\right)$ by induction on $i$.

For $i=1$, in every packing there is exactly one bin with one item, and this packing is a staircase packing. For a given value of $i, n=i(i+1) / 2$. We consider a subset of $n^{\prime}=n-i=i(i-1) / 2$ items. By the induction hypothesis it is possible to obtain a packing such that for any $1 \leq \eta \leq i-1$ there is a bin with $\eta$ items. Considering the complete set of $n$ items, we get that for any $2 \leq \eta \leq i-1$ there is a bin with $\eta$ items, and additionally there are $i+1$ bins, each with a single item. By the induction hypothesis, this packing is obtained in $i(i-1)(i-2) / 6$ steps. Let $B_{\eta}$ denote a specific bin with $\eta$ items for $1 \leq \eta \leq i-1$, where the bin $B_{1}$ is chosen arbitrarily. The $i$ other items packed in dedicated bins are called free items. For $k=1, \ldots, i-1$, the $k$-th free item is moved from its bin, to the bins $B_{1}, B_{2}, \ldots$, $B_{i-k}$, in this order. $B_{i-k}$ will now contain $i-k+1$ items and will not be used again in this process. After all these steps, $B_{\eta}$ (for $1 \leq \eta \leq i-1$ ) will contain $\eta+1$ items. The $i$-th free item remains packed in its own bin, so as a result, for any $1 \leq \eta \leq i$ there is a bin with $\eta$ items. The number of additional steps for the free items (the steps that are applied after the bins $B_{\eta}$ are created using the induction hypothesis) is

$$
\sum_{k=1}^{i-1}(i-k)=i(i-1) / 2,
$$

as the number of steps for the $k$ th free item is $i-k$. The total number of steps is therefore

$$
\frac{i(i-1)(i-2)}{6}+\frac{i(i-1)}{2}=\frac{i(i+1)(i-1)}{6} .
$$

To show the claim for the case for $j \neq 0$ (and $i \geq 2$ ), we use the claim that was proved for $j=0$. Assume that $n=i(i+1) / 2-j$ where $0<j<i$. In this case, first we create a staircase packing of a subset of $n^{\prime}=i(i-1) / 2$ items, leaving $i-j$ free items. For $k=1, \ldots, i-j$, the $k$-th free item is moved from its bin, to the bins $B_{1}, B_{2}, \ldots, B_{i-k}$, in this order. The bin $B_{i-k}$ will contain $i-k+1$ items as a result and will not be used for later steps. After this is done for $i-j$ items, $B_{\eta}$ will contain $\eta+1$ items for $j \leq \eta \leq i-1$, and for $1 \leq \eta \leq j-1, B_{\eta}$ still contains $\eta$ items. Thus, for every $1 \leq \eta \leq i, \eta \neq j$, there is exactly one bin with $\eta$ items and this is exactly a staircase packing as required. The number of additional steps 
(after the bins $B_{\eta}$ are created using the claim for $j=0$ ) is

$$
\sum_{k=1}^{i-j}(i-k)=i(i-1) / 2-j(j-1) / 2 .
$$

The total number of steps is

$$
\frac{i(i+1)(i-1)}{6}-\frac{j(j-1)}{2} .
$$

Once a staircase packing is achieved, we show that it is possible to reach a packing where all items are packed in one bin together using exactly $i(i+1)(i-$ $1) / 6-i j+j(j+1) / 2$ steps. We define a phase as follows. In the beginning of a phase there are bins with different numbers of items. Let

$$
J=\left\{j_{1}<j_{2}<\cdots<j_{|J|}\right\}
$$

be the set of numbers of items before some phase, and let the bin $B_{\eta}$ for $\eta \in J$ be the bin with $\eta$ items. If $|J|>1$, we repeatedly take an item from $B_{j_{1}}$, and move it to $B_{j_{2}}$ then to $B_{j_{3}}$ and so forth until it reaches $B_{j_{|J|}}$. A phase ends when all items of $B_{j_{1}}$ were moved. If $j=0$, then initially $J=\{1, \ldots, i\}$, there are $i-1$ phases, and the number of steps in all phases is

$$
\sum_{k=1}^{i-1} k(i-k)=\frac{i^{2}(i-1)}{2}-\frac{(i-1) i(2 i-1)}{6}=\frac{i(i-1)(i+1)}{6} .
$$

Otherwise, initially $J=\{1, \ldots, i\}-\{j\}$, there are $i-2$ phases, and the number of steps is

$$
\begin{gathered}
\sum_{k=1}^{j-1} k(i-1-k)+\sum_{k=j+1}^{i-1} k(i-k)=\sum_{k=1}^{i-1} k(i-k)-\sum_{k=1}^{j-1} k-j(i-j) \\
=\frac{i^{2}(i-1)}{2}-\frac{i(i-1)(2 i-1)}{6}-\frac{j(j-1)}{2}-j(i-j)=\frac{i(i+1)(i-1)}{6}-i j+\frac{j}{2}+\frac{j^{2}}{2} .
\end{gathered}
$$

The total number of steps is therefore

$$
\frac{i(i+1)(i-1)}{6}-\frac{j(j-1)}{2}+\frac{i(i-1)(i+1)}{6}+\frac{j}{2}-i j+\frac{j^{2}}{2}=\frac{i(i+1)(i-1)}{3}+j-i j=\nabla_{i, j} .
$$

Next, we prove the main result of this paper.

Theorem 1. The number of steps until convergence is at most

$$
\nabla_{i, j}=\frac{i(i+1)(i-1)}{3}+j-i j=\Theta\left(n^{\frac{3}{2}}\right)
$$

and there exists an input of $n$ items where this bound can be achieved. 
Proof. The lower bound was proved in the previous lemma. For the upper bound, consider an input $I$ of $n=i(i+1) / 2-j$ items for $0 \leq j \leq i-1$, an initial configuration and a sequence of moves. Let $p_{\min }$ denote the smallest item size in $I$. Let $\varepsilon=\min \left\{p_{\min }, 1 / n\right\}$, and let $I^{\prime}$ be the input where $s_{t}=\varepsilon$ for $1 \leq t \leq n$. For the input $I^{\prime}$ there cannot be invalid moves, since all items can be packed into one bin.

Lemma 2. The initial configuration and the sequence of moves of $I$ are valid for $I^{\prime}$ as well.

Proof. Since no item size was increased, all configurations of $I$ are valid for $I^{\prime}$. Since the cost of an item in a packing depends only on numbers of items in its bin and not on their sizes, modifying the sizes may only increase sets of beneficial deviations, that is, every move that was beneficial and possible for $I$ remains such for $I^{\prime}$ and the sequence of moves is still valid.

In what follows, we will consider only sequences of moves for $I^{\prime}$. In particular, we consider only sequences with a maximum number of moves. Such a sequence must exist since from the results of [14] every sequence of moves has a finite length.

Lemma 3. Every sequence with a maximum number of moves starts with the configuration where every item is packed in a separate bin, and ends with the configuration that all items are packed in one bin.

Proof. Consider a sequence of $\ell$ moves. Assume that there is a bin $B$ with $k \geq 2$ items in the initial configuration, and let $\phi \in B$. Modify the configuration such that instead of $B$ the starting configuration has the two bins $B \backslash\{\phi\}$ and $\{\phi\}$ (other bins remain unchanged). Next, add a step in the beginning of the sequence of moves where $\phi$ moves to join the items of $B \backslash\{\phi\}$. This is an improving step since $\phi$ reduces its cost from 1 to $\frac{1}{k}$. This results in a sequence of $\ell+1$ steps, which contradicts maximality.

Next, assume that after the sequence of moves there are at least two non-empty bins, containing $k_{1}$ and $k_{2}$ items respectively, where $k_{1} \leq k_{2}$. Let $\psi$ be an item packed in the first bin. Add a move of $\psi$ to the second bin in the end of the sequence. This is an improving step since $\psi$ reduces its cost from $\frac{1}{k_{1}}$ to $\frac{1}{k_{2}+1} \leq \frac{1}{k_{1}+1}<\frac{1}{k_{1}}$. This results in a sequence of $\ell+1$ steps, which contradicts maximality.

Let $k>0$ be an integer. We define a level $k$ small step to be a move where an item moves from a $k$-bin to another $k$-bin. A step is called a small step if there is an integer $k$ such that the step is a level $k$ small step. Given the set of sequences of steps of maximum length we focus on sequences where the maximum length prefix of small steps has maximum length.

Lemma 4. Assume that after a prefix of the sequence of steps is applied there are at least two $k$-bins. Then the first step in the remainder of the sequence of steps involving a $k$-bin is a level $k$ small step. 
Proof. Assume by contradiction that there is no level $k$ small step in the remaining part of the sequence. Since the sequence of steps terminates only when all items are packed in one bin, there is at least one item in the union of the $k$-bins that will perform a move (in fact, all the items of all the $k$-bins except for possibly one such bin will do that). Consider the first step after the current configuration was reached that involves a $k$-bin (either an item moving to the bin or moving out of it).

There are two possible moves. If an item $\psi$ moves from a $k$-bin into a bin with $k^{\prime}>k$ items, we modify the sequence as follows. First $\psi$ moves to another $k$-bin, and then it moves to the bin with $k^{\prime}$ items. The second step is still beneficial for $\psi$ since in the second step it moves from a $(k+1)$-bin to a bin with $k^{\prime} \geq k+1$ items. This modification augments the length of the sequence by 1 , which contradicts maximality.

If an item $\phi$ moves from a bin with $\tilde{k}<k$ items to one of the $k$-bins, we modify the sequence as follows. First choose an arbitrary item from one of the $k$-bins and move it to another $k$-bin. Then, move $\phi$ to the bin out of which the item was just moved (which now has $k-1$ items). This last move is beneficial since $\tilde{k} \leq k-1$. This modification augments the length of the sequence by 1 , which contradicts maximality.

Lemma 5. Consider a maximum length prefix of small steps. After this prefix is performed, every bin has a different number of items.

Proof. Assume by contradiction that at this time there are two $k$-bins. Using Lemma 4 , there will be a level $k$ small step later in the sequence, which will be the first move that involves $k$-bins. Since all items are identical, it is possible to perform such a step immediately instead of at a later time. This does not change the number of steps in the sequence, and it increases the length of the maximum length prefix of small steps, which contradicts maximality of the prefix (out of sequences of maximum length).

Lemma 6. Consider the maximum length prefix of small steps. After this prefix is performed, there is one bin of each number of items in $\{1,2, \ldots, i\} \backslash\{j\}$, that is, a staircase packing is created.

Proof. We prove an invariant that is kept as long as only small steps are done. Let $b_{k}$ be the number of bins with $k$ items, and recall that initially $b_{1}=n$ and $b_{\ell}=0$ for $0<\ell \leq n$. Assume that at a given time, $k_{m}$ is the maximum integer such that $b_{k_{m}}>0$. We say that a number $1 \leq k \leq k_{m}-1$ is bad if $b_{k}=0$, and otherwise it is good. That is, a number $k$ is bad if there are no $k$-bins, but there exists at least one $(k+1)^{+}$-bin. If $b_{k} \geq 2$ then we say that $k$ is very good. Two bad numbers are called consecutive bad numbers if all numbers between them are good, that is, if $k_{1}$ and $k_{2}$ such that $k_{1}<k_{2}<k_{m}$ are both bad $\left(b_{k_{1}}=0\right.$ and $\left.b_{k_{2}}=0\right)$, and for all $k^{\prime}$ such that $k_{1}<k^{\prime}<k_{2}, b_{k^{\prime}}>0$. 
The invariant is as follows. For every pair of consecutive bad numbers $k_{1}, k_{2}$, where $1 \leq k_{1}<k_{2}<k_{m}$, there exists a number $\tilde{k}$, where $k_{1}<\tilde{k}<k_{2}$, such that $\tilde{k}$ is very good.

Initially, $k_{m}=1$, thus there are no bad numbers, and the invariant holds trivially. Recall that we only analyze small steps and consider the change resulting from a single level $k$ small step. Every level $k$ small step implies that before this step there are at least two $k$-bins and so $k$ is very good.

Note that $k$ is the only number that can become bad as a result of a level $k$ small step. Moreover, if $k=k_{m}$, then the value $k_{m}$ increases by 1 . Assume first that $k$ remains very good. No bad numbers are created, and since no number stops beings very good then the invariant holds (even if some number stops being bad). If $k$ remains good, but not very good, then still no new bad numbers are created and we only need to consider the case that $k$ was the only very good number between two consecutive bad numbers. Let these two numbers be $k_{1}<k<k_{2}$. If $k_{2}>k+1$ and $k_{1}<k-1$, then the numbers of $k_{1}$-bins and $k_{2}$-bins are unchanged (that is, these numbers remain zero) and the numbers $k_{1}, k_{2}$ remain consecutive bad numbers between which we need to show that a very good number exists after the step. Since $k+1$ was good, as a result of the move $b_{k+1} \geq 2$, and since $k_{1}<k+1<k_{2}$, there is a very good number between $k_{1}$ and $k_{2}$, as required. If $k_{1}=k-1$ but $k_{2}>k+1$ then $k_{1}$ becomes good. If $k_{1}$ was the minimum bad number then we are done. Otherwise, let $k_{3}<k_{1}$ be a bad number such that $k_{3}$ and $k_{1}$ were consecutive bad numbers. We now have that $k_{3}$ and $k_{2}$ are consecutive bad numbers and $b_{k+1} \geq 2$ so $k+1$ is a very good number between them. If $k_{1}<k-1$ but $k_{2}=k+1$ then $k_{2}$ becomes good. If $k_{2}$ was the maximum bad number then we are done. Otherwise, let $k_{4}>k_{2}$ be a bad number such that $k_{2}$ and $k_{4}$ were consecutive bad numbers. We now have that $k_{3}$ and $k_{4}$ are consecutive bad numbers and $b_{k-1} \geq 2$ so $k-1$ is a very good number between them. Finally, if both $k_{1}=k-1$ and $k_{2}=k+1$ hold, then the only case of interest is when $k_{1}$ was not the minimum bad number and $k_{2}$ was not the maximum bad number. We let $k_{3}<k_{1}$ be a bad number such that $k_{3}$ and $k_{1}$ were consecutive bad numbers, and let $k_{4}>k_{2}$ be a bad number such that $k_{4}$ and $k_{2}$ were consecutive bad numbers. Now $k_{3}$ and $k_{4}$ are consecutive bad numbers. There is a very good number in $\left(k_{3}, k_{1}\right)$ which is now a very good number between $k_{3}$ and $k_{4}$.

Finally, we consider the case where $k$ becomes bad. If there previously was a bad number $k_{2}$ such that $k_{2}>k$, we distinguish two cases. If $k_{2}>k+1$, then $k$ and $k_{2}$ becomes a consecutive bad pair of numbers, and $k+1$ becomes a very good number between them. Otherwise, $k_{2}=k+1$ becomes good. If $k_{2}$ was the maximum bad number then we are done, and otherwise, let $k_{4}>k_{2}$ be such that $k_{2}$ and $k_{4}$ were consecutive bad numbers. Instead, $k$ and $k_{4}$ are now consecutive bad numbers, and the very good number between them is the same one which was very good between $k_{2}$ and $k_{4}$. The proof is symmetric for the case that there previously was a bad number $k_{1}$ such that $k_{1}<k$.

To complete the proof, consider the configuration after the (maximum length) prefix of small steps. Since every bin has a different number of items, there are no very good numbers, and hence, by the invariant, there is at most one bad number. 
If there exists a bin with at least $i+1$ items, and there is just one bad number, then there are at least $(i+1)(i+2) / 2-i=i(i+1) / 2+1>n$ items. If there is no bin with at least $i$ items, then there are at most $i(i-1) / 2<n$ items. Thus, there is a bin with $i$ items, and since there is at most one bad number, the bad number must be $j$ if $j \neq 0$, and otherwise there is no bad number. Therefore, the packing at this time is a staircase packing.

Lemma 7. The number of steps in the (maximum length) prefix of small steps is at most

$$
\frac{i(i+1)(i-1)}{6}-\frac{j(j-1)}{2} .
$$

Proof. We use the potential function as in [14] which is the sum of squares of number of items in the bins. In the beginning every item is in a dedicated bin, so the potential is equal to $n=i(i+1) / 2-j$. Consider a level $k$ small step. The potential function increases by exactly 2 in this step, since the only change is that instead of two $k$-bins, there is a $(k-1)$-bin a $(k+1)$-bin, and the increase in the potential is exactly

$$
(k+1)^{2}+(k-1)^{2}-2 k^{2}=2 .
$$

Since a staircase packing is achieved in the (maximum length) prefix of small steps, the value of the potential after this prefix is

$$
\sum_{k=1}^{i} k^{2}-j^{2}=\frac{i(i+1)(2 i+1)}{6}-j^{2} .
$$

Thus, the number of steps cannot exceed half the difference between the final potential and the initial potential, which is

$$
\left(\frac{i(i+1)(2 i+1)}{6}-j^{2}-\left(\frac{i(i+1)}{2}-j\right)\right) / 2=\frac{i(i+1)(i-1)}{6}-\frac{j(j-1)}{2} .
$$

Lemma 8. The number of steps in the remainder of the sequence after the (maximum length) prefix of small steps is at most

$$
\frac{i(i+1)(i-1)}{6}-i j+\frac{j(j+1)}{2} .
$$

Proof. In this case we define a different potential function. Sort the bins in nonincreasing order according to numbers of items. Let the index of an item be the index of the bin into which it is packed. The potential of a packing is sum of indices of items.

The potential is clearly positive at all times. The final potential is $n$, since all items are packed in one bin. Consider a step in which an item moves from a $k_{1}$-bin $B_{v}$ to a $k_{2}$-bin $B_{u}$ (where $k_{2} \geq k_{1}$ ). Since all items are identical, we assume that $B_{v}$ is the $k_{1}$-bin of maximum index, and $B_{u}$ is the $k_{2}$ bin of minimum index. This 
holds even if $k_{1}=k_{2}$, since in this case there are at least two bins with this number of items. Since the bins are sorted by non-increasing order according to numbers of items we have $v>u$. As a result of the move, $B_{v}$ now has $k_{1}-1$ items, and $B_{u}$ now has $k_{2}+1$ items. By definition, if $u>1$ then $B_{u-1}$ has at least $k_{2}+1$ items. Similarly, if $B_{v+1}$ exists then it has at most $k_{1}-1$ items, so the sorted order is still valid. The change in the potential in this step is the change in the index of the bin of the moving item, which is $v-u \geq 1$.

If $j=0$, then the potential before the remainder of the sequence of moves is performed is

$$
\sum_{k=1}^{i} k(i-k+1)=\frac{i(i+1)^{2}}{2}-\frac{i(i+1)(2 i+1)}{6}=\frac{i(i+1)(i+2)}{6}
$$

while $n=\frac{i(i+1)}{2}$, so the number of steps is at most

$$
\frac{i(i+1)(i+2)}{6}-\frac{i(i+1)}{2}=\frac{i(i+1)(i-1)}{6} .
$$

If $j>0$, then the potential before the remainder of the sequence of moves is performed is

$$
\begin{gathered}
\sum_{k=1}^{i-j} k(i-k+1)+\sum_{k=i-j+1}^{i-1} k(i-k)=i \sum_{k=1}^{i-1} k+\sum_{k=1}^{i-j} k-\sum_{k=1}^{i-1} k^{2} \\
=\frac{i^{2}(i-1)}{2}+\frac{(i-j)(i-j+1)}{2}-\frac{i(i-1)(2 i-1)}{6} \\
=\frac{i(i-1)(i+1)}{6}+\frac{i^{2}}{2}+\frac{j^{2}}{2}-i j+\frac{i}{2}-\frac{j}{2} .
\end{gathered}
$$

In each step the function decreases by at least 1 , so the number of steps is at most

$\frac{i(i-1)(i+1)}{6}+\frac{i^{2}}{2}+\frac{j^{2}}{2}-i j+\frac{i}{2}-\frac{j}{2}-\frac{i(i+1)}{2}+j=\frac{i(i+1)(i-1)}{6}-i j+\frac{j}{2}+\frac{j^{2}}{2}$.

Taking the sum of the maximum number of steps in the prefix (till the last small step of the maximum length prefix of small steps) with that of the remainder we get

$\frac{i(i+1)(i-1)}{6}-\frac{j(j-1)}{2}+\frac{i(i+1)(i-1)}{6}-i j+\frac{j(j+1)}{2}=\frac{i(i+1)(i-1)}{3}+j-i j=\nabla_{i, j}$. 


\section{References}

[1] R. Adar and L. Epstein. Selfish bin packing with cardinality constraints. Theoretical Computer Science, 495:66-80, 2013.

[2] E. Anshelevich, A. Dasgupta, J. M. Kleinberg, É. Tardos, T. Wexler, and T. Roughgarden. The price of stability for network design with fair cost allocation. SIAM Journal on Computing, 38(4):1602-1623, 2008.

[3] V. Bilò. On the packing of selfish items. In Proc. of the 20th International Parallel and Distributed Processing Symposium (IPDPS'06). IEEE, 2006. 9 pages.

[4] E. G. Coffman, M. R. Garey, and D. S. Johnson. Approximation algorithms for bin packing: A survey. In D. Hochbaum, editor, Approximation algorithms. PWS Publishing Company, 1997.

[5] E. Coffman Jr. and J. Csirik. Performance guarantees for one-dimensional bin packing. In T. F. Gonzalez, editor, Handbook of Approximation Algorithms and Metaheuristics, chapter 32, pages (32-1)-(32-18). Chapman \& Hall/Crc, 2007.

[6] J. Csirik and G. J. Woeginger. On-line packing and covering problems. In A. Fiat and G. J. Woeginger, editors, Online Algorithms: The State of the Art, pages 147-177, 1998.

[7] Gy. Dósa and L. Epstein. Generalized selfish bin packing. CoRR, abs/1202.4080, 2012.

[8] Gy. Dósa and L. Epstein. The convergence time for selfish bin packing. In Proc. of The rth International Symposium on Algorithmic Game Theory (SAGT'14), 37-48, 2014.

[9] L. Epstein and E. Kleiman. Selfish bin packing. Algorithmica, 60(2):368-394, 2011.

[10] L. Epstein, E. Kleiman, and J. Mestre. Parametric packing of selfish items and the Subset Sum algorithm. Algorithmica, 74(1): 177-207, 2016.

[11] E. Even-Dar, A. Kesselman, and Y. Mansour. Convergence time to Nash equilibrium in load balancing. ACM Transactions on Algorithms, 3(3):32, 2007.

[12] U. Faigle and W. Kern. On some approximately balanced combinatorial cooperative games. Mathematical Methods of Operations Research 38(2), 141-152, 1993.

[13] U. Faigle and W. Kern Approximate core allocation for binpacking games. SIAM Journal on Discrete Mathematics, 11(3):387-399, 1998. 
[14] R. Ma, Gy. Dósa, X. Han, H.-F. Ting, D. Ye, and Y. Zhang. A note on a selfish bin packing problem. Journal of Global Optimization, 56(4), 1457-1462, 2013.

[15] R. Holzman and N. Law-Yone. Strong equilibrium in congestion games. Games and Economic Behavior, 21(1-2):85101, 1997.

[16] S. Ieong, B. McGrew, E. Nudelman, Y. Shoham, and Q. Sun. Fast and compact: A simple class of congestion games, In Proceedings of the 20th National Conference on Artificial Intelligence, pages 489-494, 2005.

[17] D. S. Johnson, A. Demers, J. D. Ullman, M. R. Garey, and R. L. Graham. Worst-case performance bounds for simple one-dimensional packing algorithms. SIAM Journal on Computing, 3:256-278, 1974.

[18] W. Kern and X. Qiu. Integrality gap analysis for bin packing games. Operations Research Letters, 40(5):360-363, 2012.

[19] F. K. Miyazawa and A. L. Vignatti. Convergence time to Nash equilibrium in selfish bin packing. Electronic Notes in Discrete Mathematics, 35:151-156, 2009.

[20] F. K. Miyazawa and A. L. Vignatti. Bounds on the convergence time of distributed selfish bin packing. International Journal of Foundations of Computer Science, 22(3):565-582, 2011.

[21] J. Nash. Non-cooperative games. Annals of Mathematics, 54(2):286-295, 1951.

[22] G. Yu and G. Zhang. Bin packing of selfish items. In The 4th International Workshop on Internet and Network Economics (WINE'08), pages 446-453, 2008. 